\title{
Topology Optimization considering Additive Manufacturing constraints in an industrial context
}

\author{
Sourena MOOSAVI ${ }^{\mathrm{a}, *}$, Dominique CHAMORET ${ }^{\mathrm{b}}$, Salima TIE BI ${ }^{\mathrm{b}}$, Naoual \\ SABKHI $^{\mathrm{a}}$, Yannick CULNARD ${ }^{\mathrm{a}}$ \\ ${ }^{a}$ RED Department, Altran Technologies, 11 Rue de la découverte 90000 Belfort France. \\ name.lastname@altran.com \\ ${ }^{b}$ Laboratoire Interdisciplinaire Carnot de Bourgogne, UMR CNRS 6303 / Univ. \\ Bourgogne Franche Comté (UBFC), UTBM, 90000 Belfort, France. \\ name.lastname@utbm.fr
}

\begin{abstract}
Manufacturing-oriented Topology Optimization (TO) is an active field of research both in academic and in industrial world. On one hand, the potential offered by topology optimization as a powerful design method is undeniable in improving the structural performance in different areas of engineering. On the other hand, Additive Manufacturing (AM) technology provides numerous industrial mechanisms of manufacturing and producing complex structure where classical methods fail to deliver an outcome.

Merging both branches of TO and AM together introduces encouraging results in terms of designing optimized structures showing an acceptable mechanical response. In our contribution, we present a topology optimization model developed for additive manufacturing design. Using an innovative meshing algorithm, we particularly showed how the topology optimization could be implemented in order to consider the constraints of additive manufacturing specifically the printability of the parts with overhang angle.

The design of the self-supporting structures is a challenging part of the additive manufacturing process, without which the process becomes tedious in terms of time, efficiency and financial resources. Therefore, a continuous effort for a better understanding of such issue is justified. In this paper, we suggest an improvement on the work carried out by Langelaar in this context. The model used in this study is a more complicated geometry compared to
\end{abstract}

${ }^{*}$ Corresponding author 
the model used by Langelaar. While the model used by Langelaar is a simple academic model, our work is based on a complex industrial geometry. The Optimality Criteria (OC) was exploited as the TO method in our work. The novelty of our work lies particularly in utilising the unstructured mesh, which, is a first attempt in using such grids in this context. An innovative numerical algorithm is designed specifically for taking into account the critical overhang design in order to avoid support structures. It is worth mentioning that the numerical results obtained in our research work are the outcome of an in house code developed in collaboration between Altran Technologies and Université de Technologie de Belfort-Montbéliard.

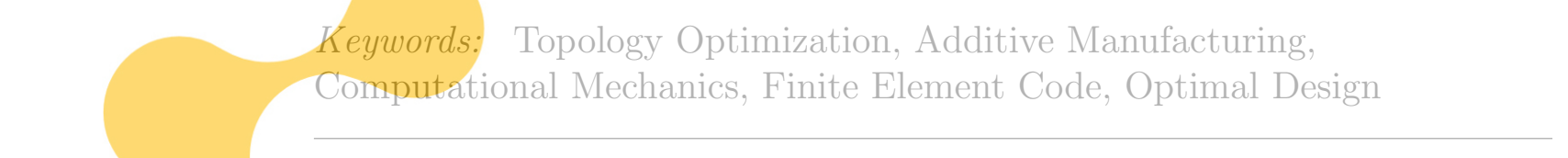

\section{Introduction}
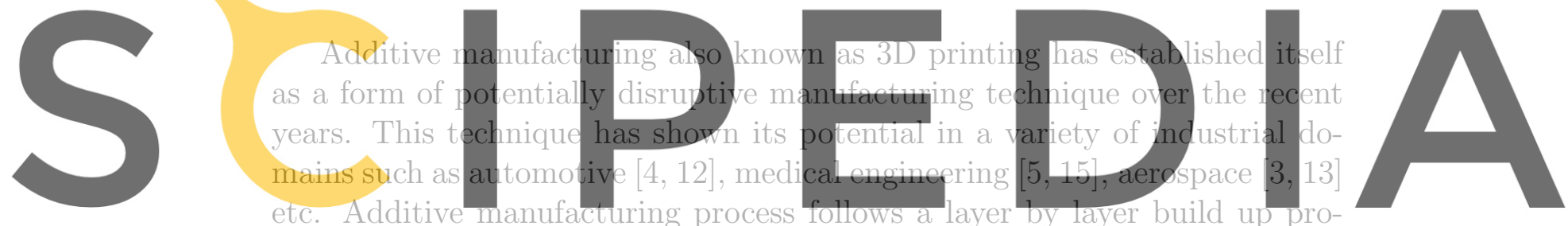

cess which gives an entirely different nature to this technique in comparison

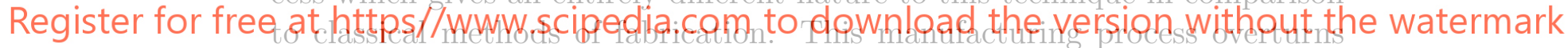

the traditional manufacturing method that is the subtractive manufactur-

ing, that makes the fabrication of new and complex geometrical features possible. The different build process offered by additive manufacturing removes the geometry complexity restriction faced in classical methods and eventually the efficiency and cost of fabrication is going to be unaffected by this limitation [8]. This latter characteristic provides an extended manufaturability freedom which makes additive manufacturing a great candidate for exploiting the free-form designs put forward by topology optimization [10].

Topology optimization can be defined as a free-form material distribution scheme that aims at seeking optimal material distribution for obtaining enhanced mechanical or multi-physics performances. However, one of the main challenges of topology optimization nowadays is the manufacturability of the obtained optimized designs. As mentioned the geometries proposed by topology optimization methods are best exploited by additive manufacturing although there remains some limitations to this unique fabrication technique 
that need to be addressed in topology optimization algorithms. Some of the problems have been tackled through the past two decades. Topology optimization with material anisotropy was targeted by [1], self-support structure design was discussed by [9]. One of the greatest challenges encountered in manufacturing the structures proposed by topology optimization methods is the overhang angle that needs special treatment during the process of fabrication by additive manufacturing. To overcome this problem, there is a need to discover the best procedure in introducing this constraint within the topology optimization algorithm to achieve better results in terms of manufacturability. We addressed the issue in this research paper by introducing a new algorithm that removes need of so called support structures that are partly used for tackling the overhang problems.

\section{Problem definition and context}
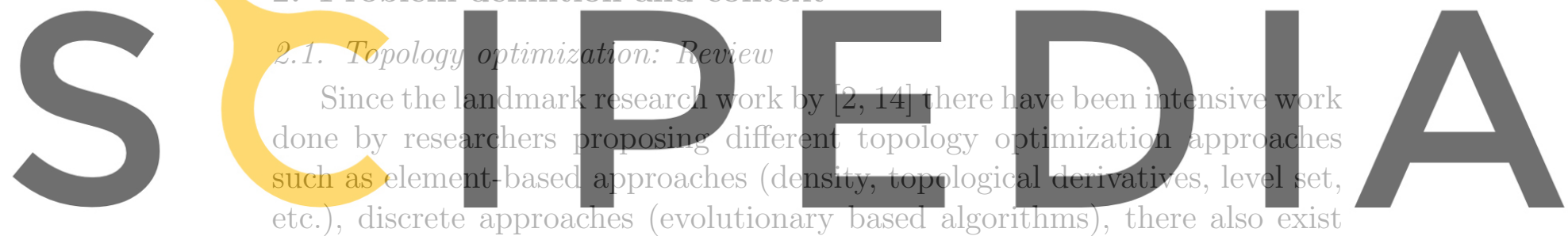

Register for free at https. www scipediacom to downjoad the version without the watermark

We use the optimality criteria method (OC) for topology optimization, which is one of the most prominent gradient-based mathematical optimization algorithms. This method was used by [14] in a 99-line MATLAB code where he discussed optimizing an MBB (Messerschmitt-Bolkow-Blohm) problem. In general, optimization algorithms are associated with the Finite Element Method as shown in Figure 1 thus, we follow the same logic in this paper. The following mathematical representation summarizes the topology optimization problem discussed in current work. That is maximizing the global structural static stiffness of a structure. This is equivalent to minimize the compliance (strain energy) :

$$
\begin{aligned}
\text { Minimize : } & C(X)=\mathbf{U}^{T} \mathbf{K U} \text { with } X=\left(\rho_{1}, \rho_{2}, \cdots, \rho_{n}\right) \\
\text { Subjected to : } & \frac{V(X)}{V_{0}}=f
\end{aligned}
$$

where 


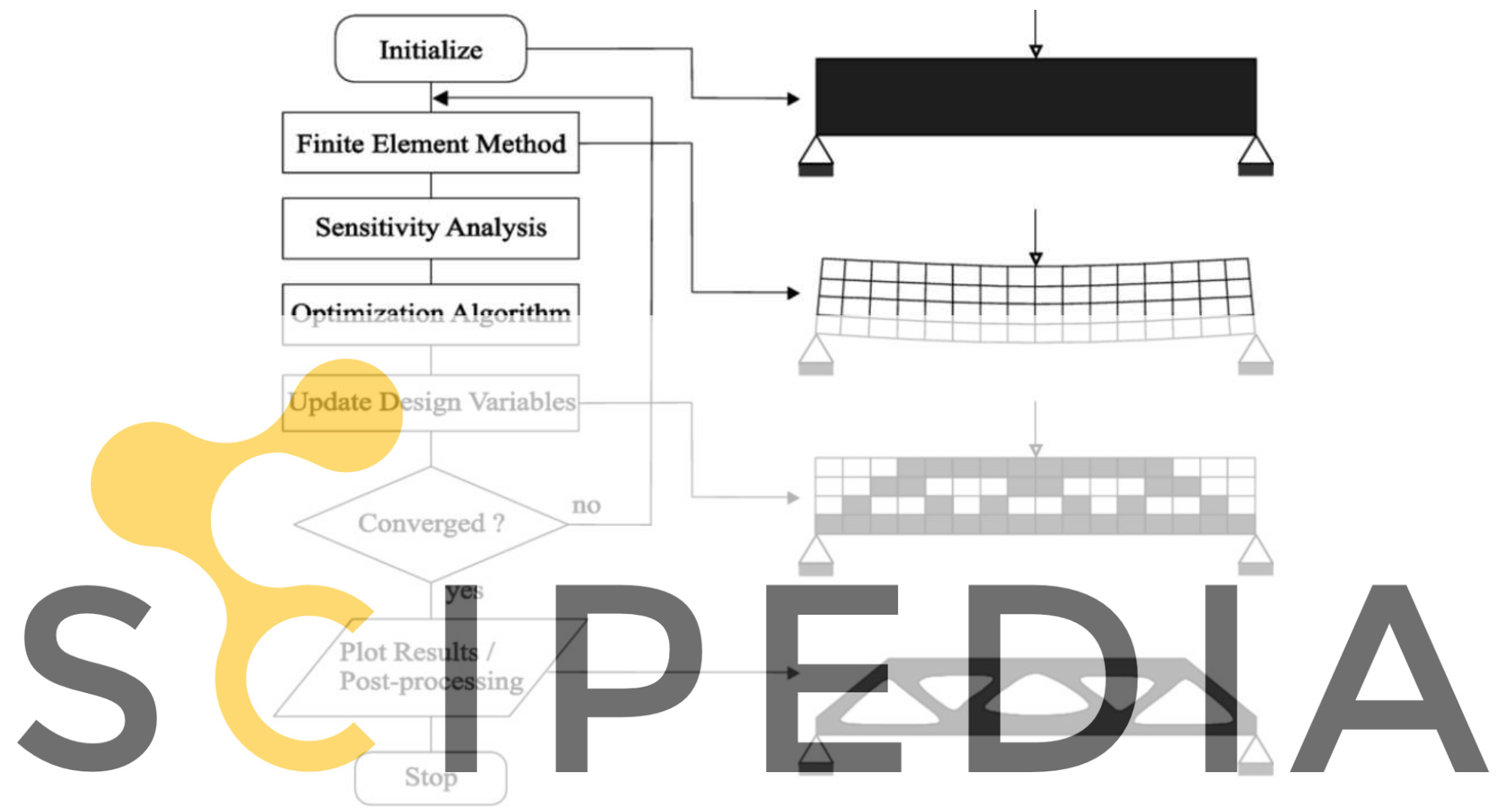

Register for free at https./Www. scipedia.com to download the version without the watermark

- $C$ is the compliance (objective function),

- $\mathbf{U}$ and $\mathbf{K}$ are the global displacement vector and global stiffness matrix respectively,

- $V(X)$ and $V_{0}$ are the material volume and design volume respectively,

- $f$ is the prescribed volume fraction (maximum volume fraction of material remaining),

- $X$ is the vector of design variables ( $\rho_{i}$ is the density of element $i$ ).

\subsection{AM Contrainst : overhang angle}

In most additive manufacturing processes in order to have successfully built overhang components support structures need to be integrated in dif- 
ferent stages of the process. The disadvantages of the necessity for support structures is an issue that is an ongoing field of research where solutions to alleviate the negative effects of such structures are being sought. In majority of optimization cases, the proposed model by optimization algorithm tends to form the overhanging structure which technically requires a support structure for production purpose in additive manufacturing. Various researchers proposed different approaches to target this issue. For instance support slimming seeks to minimize the amount of material used in support structures.

In an ideal case the reduction of support structures to zero is preferred. In the following section we discuss our proposed algorithm integrated in the optimization iteration that provides the designers to avoid having any support structures at all.

\subsection{Proposed approach}

To overcome the problem of support structures, there is a need to dis-
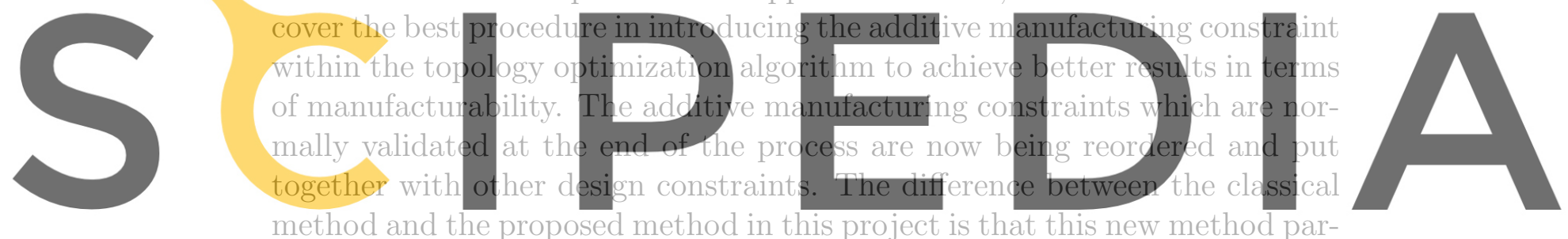

Register for free allelizes the two validations which refer to ad ditivg manufacturing canstraint

and other constraints at the same time for making the topology optimiza-

tion algorithm more efficient by shortening the time and reduce the overall

cost (Figure 2). Our method is an extension of the method introduced by Langelaar. In his method Langelaar $[6,7]$ included a simplified additive manufacturing process model in the problem formulation, where the overhang angle restriction that applies to the targeted additive manufacturing processes was rigorously enforced at every step of the optimization process. The mechanism functions by means of a layer-wise applied spatial filter, simulating the printing of the part. The novelty of our work is computing the gravity center of each element, once the gravity center of each element is defined the printing layers are defined according to the printing direction. In addition to the mentioned criteria we define a critical angle for each element that sweeps the inferior layer elements in a conical shape. All the elements that are simultaneously included in the immediate inferior layer and whose gravity center is swept by the critical angle cone are considered by the code as the support elements. 


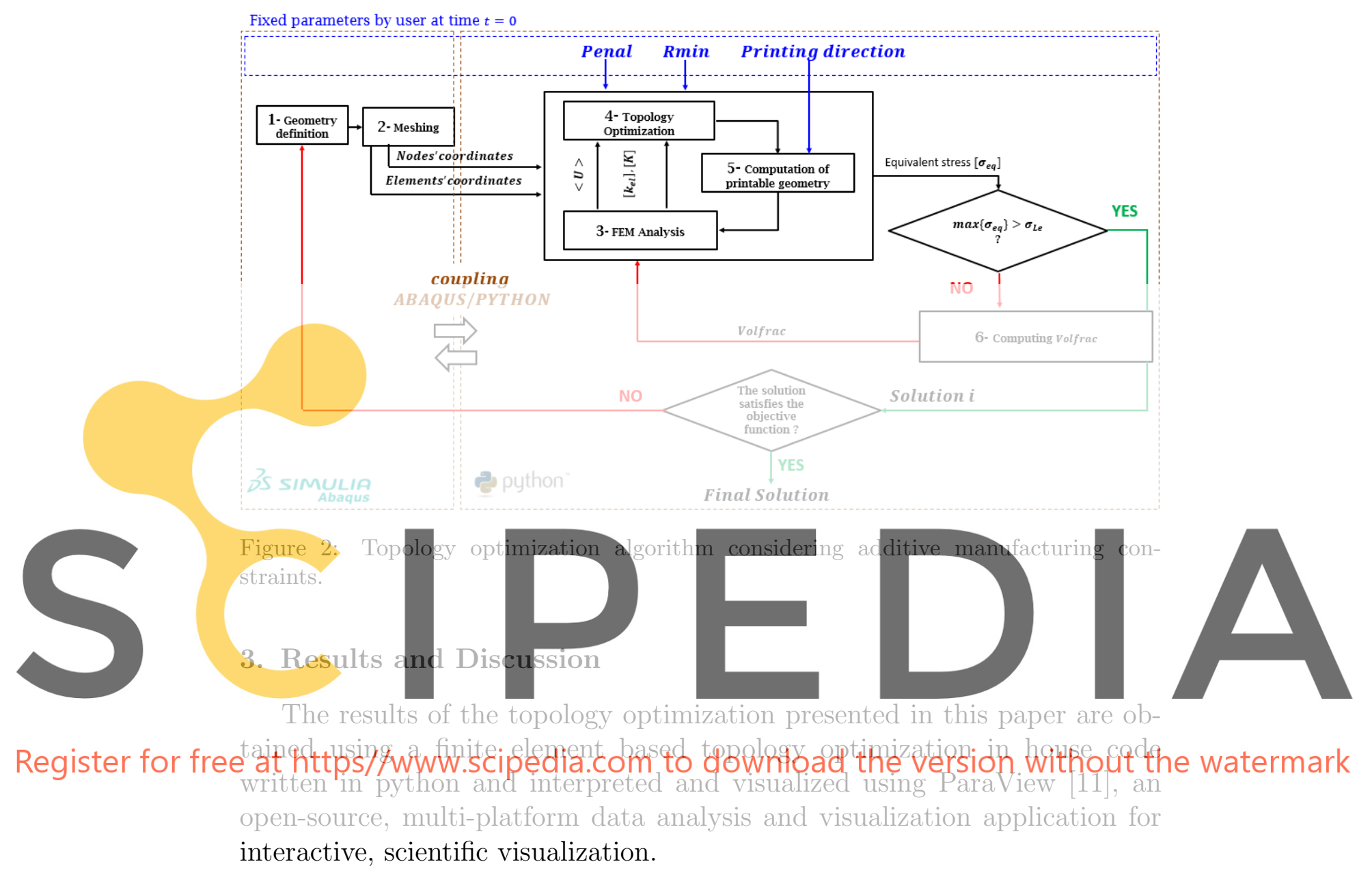

Overall our study was carried out considering two main criteria,

- Compliance minimization topology optimization with two targeted volume fractions as constraints: $0.5 V_{0}$ and $0.7 V_{0}$,

- Compliance minimization topology optimization with elastic stress limit constraint.

The Finite Element representation of the model is shown in Figure 3. Our FE model is built using 28449 of C3D8R which is a general purpose linear brick element, with reduced integration ( 1 integration point). The functional surfaces of the engine are preserved by an offset of $3 \mathrm{~mm}$ which include all 
four counter-bores, two holes on clevis arms and the entirety of the bottom surface as depicted in Figure 4. The loading condition applied to the jet engine bracket is a static linear load of $35.5 K N$ applied vertically. There is a total of 6 different simulations performed as a combination of different load cases and topology optimization criteria as shown in Table 1.
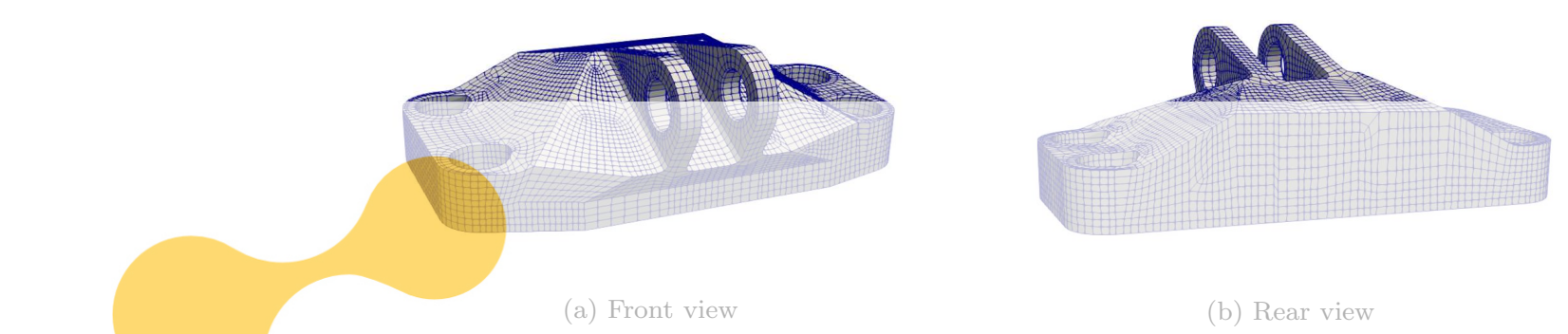

Figure 3: Finite Element rpresentation of the General Electric jet engine bracket.
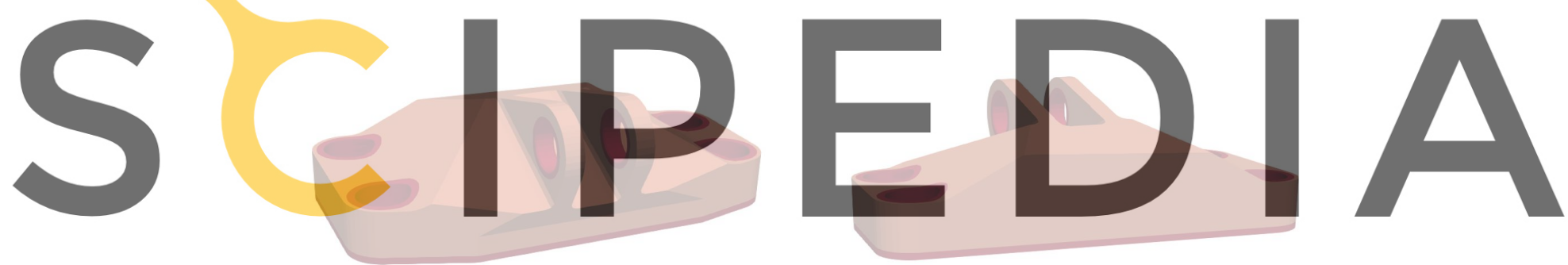

Register for free at https//wwws scipedia.com to download the version without the watermark

Figure 4: Representation of the functional surfaces on the GE jet engine bracket

Table 1: Six simulations $\left(S_{1}-S_{6}\right)$ perfoemd considering different TO criteria for two load cases.

\begin{tabular}{c|c|c|c}
\multicolumn{2}{c|}{ TO criteria } & Load case $n^{\circ} 1$ & Load case $n^{\circ} 2$ \\
\hline \multirow{2}{*}{ TO with volume fraction constraint } & 0.5 & $S_{1}$ & $S_{4}$ \\
& 0.7 & $S_{2}$ & $S_{5}$ \\
\hline Elastic stress limit constraint & $S_{3}$ & $S_{6}$ \\
\hline
\end{tabular}

Figure 5 shows the optimized jet engine bracket which is obtained using the load case $n^{\circ} 1$ which is a vertical linear static load. The topology optimization criterion considered in the process is $0.5 V_{0}$ volume fraction meaning 
that the final geometry contains only 0.5 of the initial geometry's volume. The final Von Mises stress that the piece undergoes is of 416.5 MPa magnitude.

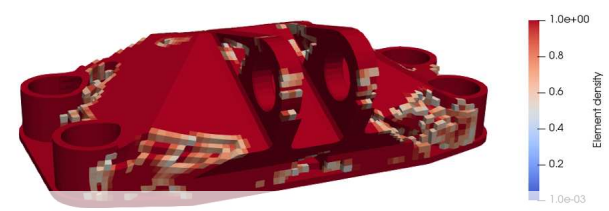

(a) Front view

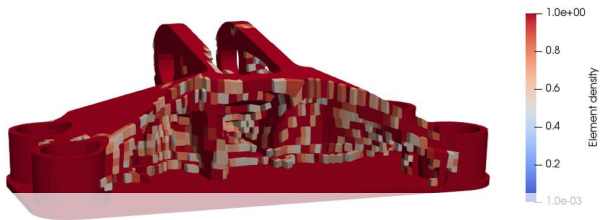

(b) Rear view

Figure 5: Optimized jet engine bracket with 0.5 of the initial volume remaining

Now considering the volume fraction of 0.7 of the the initial volume as the target we will obtain the optimized geometry as shown in Figure 6. The
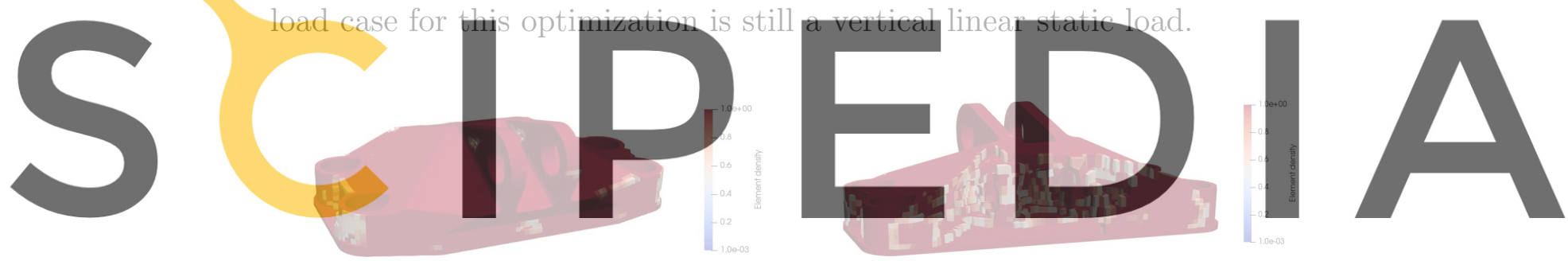

Register for free at https//wwwsicipedia.com to download thesession without the watermark

Figure 6: Optimized jet engine bracket with 0.7 of the initial volume remaining

A third type of simulation that we considered in our study is based on a final Von Mises stress threshold. The optimized piece obtained considering such criterion is not necessarily and optimized piece and it maintains 0.98 of its initial volume. 


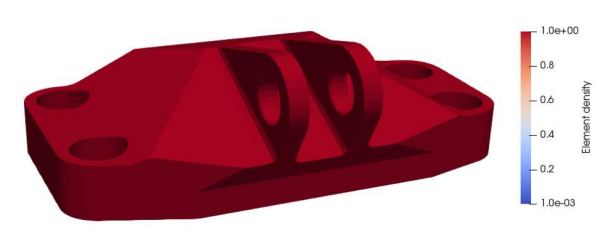

(a) Front view

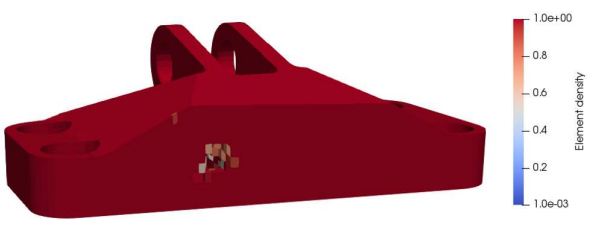

(b) Rear view

Figure 7: Optimized jet engine bracket considering elastic limit as TO criterion

\section{Conclusion}

In this study we integrated a new algorithm in to the topology optimization method OC (Optimality Criteria), enabling us to design structures ready for additive manufacturing without having to add support structures. Our algorithm allows us to reduce the structural mass without compromizing the
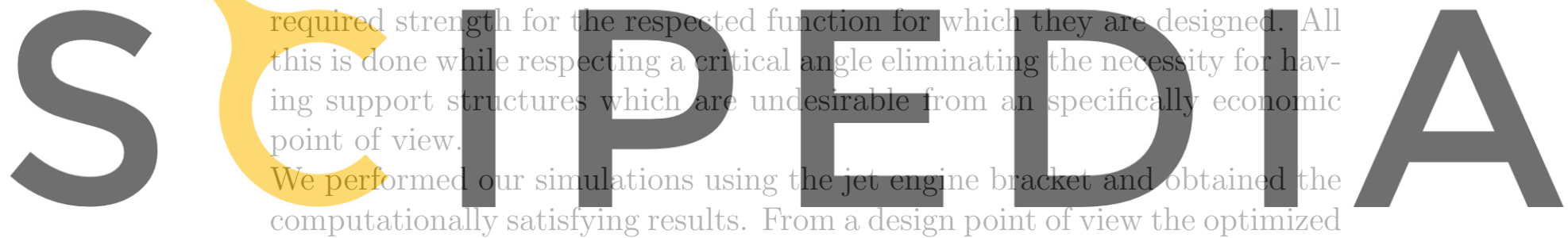

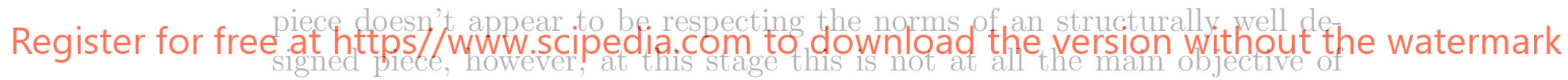

this study. A final step in order to validate our algorithm would be print-

ing our optimized piece using AM process and verify the printability of the optimized model without needing the support structures.

\section{References}

[1] Ahn S. H., Montero M., Odell D., Roundy S., Wright P. K., Anisotropic material properties of fused deposition modeling. ABS. Rapid Prototyp J 8:248-257 (2002).

[2] M. P. Bendsoe, O. Sigmund, Topology Optimization, Theory Methods and Applications, ISBN : 978-3-642-07698-5, Springer 2004.

[3] R.G. Carter, W.T. Erno, D.J. Abbott, D.H. Bruck, C.E. Wilson, G.H. Wolfe, J.B. Finkhousen, D.M. Tepper, A. Stevens, The GE aircraft en- 
gine bracket challenge: an experiment in crowdsourcing for mechanical design concepts, in: Solid Free. Fabr. Symp, 2014, pp. 1402-1411.

[4] C.H. Chuang, S. Chen, R.J. Yang, et al., Topology optimization with additive manufacturing consideration for vehicle load path development, Int. J. Numer. Methods Eng. (2017) 1434-1445.

[5] A.L. Jardini, M.A. Larosa, R.M. Filho, et al., Cranial reconstruction: 3D biomodel and custom-built implant created using additive manufacturing, J. Cranio-Maxillofacial Surg. 42 (2014) 1877-1884.

[6] M. Langelaar, Topology optimization of 3D self-supporting structures for additive manufacturing. Additive Manufacturing (2016), 12, 60-70.

[7] M. Langelaar, Combined optimization of part topology, support structure layout and build orientation for additive manufacturing, Structural
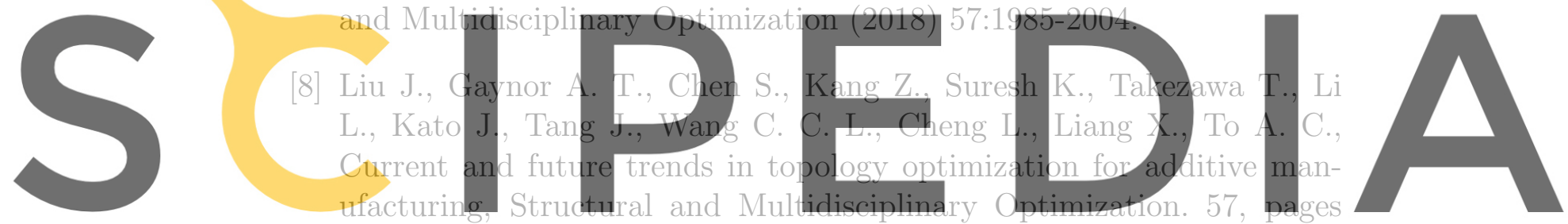

$2457-2483(2018)$.

Register for free at https//www.scipedia.com to download the version without the watermark

9] Mirzendehdel A.M., Suresh K., Support structure constrained topology optimization for additive manufacturing. Comput Aided Des 81:1-13 (2016).

[10] A. Panesar, J. Plocher, Review on design and structural optimization in additive manufacturing: Towards next-generation lightweight structures, Materials and Design 183 (2019) 108164.

[11] Ahrens, James, Geveci, Berk, Law, Charles, ParaView: An End-User Tool for Large Data Visualization, Visualization Handbook, Elsevier, 2005, ISBN-13: 978-0123875822.

[12] S.N.K. Reddy, V. Maranan, T.W. Simpson, et al., Application of topology optimization and design for additive manufacturing guidelines on an automotive component, in: Proc. ASME 2016 Int. Des. Eng. Tech. Conf. Comput. Inf. Eng. Conf, 2016, pp. 1-10. 
[13] M. Seabra, J. Azevedo, A. Araújo, et al., Selective laser melting (SLM) and topology optimization for lighter aerospace components, Procedia Struct. Integr. 1 (2016) 289-296.

[14] O. Sigmund, A 99 line topology optimization code written in Matlab, Struct. Multidiscip. Optim. 21 (2001) 120-127.

[15] S. Stübinger, I. Mosch, P. Robotti, et al., Histological and biomechanical analysis of porous additive manufactured implants made by direct metal laser sintering: a pilot study in sheep, J. Biomed. Mater. Res. - Part B Appl. Biomater. 101 (2013) 1154-1163. 